\title{
Hepatocarcinoma con componente sarcomatoide. Caso clínico
}

\author{
Héctor Losada $M^{1,2,3}$, Juan Carlos Roa ${ }^{5}$, D arío G arcía M4, \\ Juan Carlos Araya ${ }^{5}$, Luis Burgos SJ ${ }^{1,2,3}$, Jorge Silva $A^{1,2,3}$. \\ Hepatocarcinoma with sarcomatoid \\ transformation. Case report
}

Hepatocarcinoma with sarcomatoid transformation is uncommon. It presents clinically with leucocytosis and fever, resembling a liver abscess. We report a 40 year-old male that presented pain in the right upper quadrant and fever. Abdominal imaging showed an hypodense image in the liver that resembled a liver abscess. The patient was subjected to a percutaneous drainage obtaining $150 \mathrm{ml}$ of an hemorrhagic fluid whose culture was negative. The clinical picture persisted and the patient was subjected to a right hepatectomy. The pathological study of the surgical piece disclosed a hepatocarcinoma with sarcomatoid transformation. The patient was discharged sixteen days after surgery (Rev Méd Chile 2007; 135: 768-72).

(Key words: Hepatectomy; Liver abscess; Liver neoplasms)

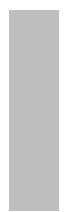

\begin{abstract}
Recibido el 2 de junio, 2006. Aceptado el 11 de septiembre, 2006.
${ }^{1}$ Departamento de Cirugía y Traumatología. Universidad de La Frontera. Temuco. Chile. ${ }^{2}$ Servicio de Cirugía. Clínica Alemana de Temuco. Temuco. Chile. ${ }^{3}$ Servicio de Cirugía. Hospital Hernán Henríquez Aravena Temuco. Chile. ${ }^{4}$ Servicio de Radiología. Clínica Alemana de Temuco. Temuco, Chile. ${ }^{5}$ Departamento de Patología. Universidad de La Frontera. Temuco. Chile.
\end{abstract}

L

os carcinomas con transformación sarcomatosa -del hígado son poco frecuentes; también se han conocido como carcinomas de células fusadas, carcinoma sarcomatoide o pseudocarcino$\mathrm{ma}^{1}$. Hay algunas comunicaciones de casos de pacientes con tumores hepáticos que se presentan con fiebre y leucocitosis simulando abscesos hepáticos, en los cuales se encontraron células neoplásicas epiteliales, que en algunos sectores tienen células fusadas en disposición trabecular, que semejan tumores sólidos ${ }^{2}$. Este tipo de pre-

Correspondencia a: Dr. Héctor Losada M. Departamento de Cirugía y Traumatología. Universidad de La Frontera. Temuco. Chile. E mail: hlosada@ufro.cl sentación es poco frecuente en Japón, con una frecuencia de $1 \%$ a $4 \%$; mientras en Sudáfrica puede alcanzar hasta $50 \% 3,4$.

El objetivo de este trabajo es comunicar el caso de un paciente con hepatocarcinoma con transformación sarcomatoide (HCTS) que se presentó con fiebre y dolor en hipocondrio derecho, simulando un absceso hepático; revisar la presentación clínica, el diagnóstico inmunohistopatológico y algunos aspectos referentes al pronóstico.

\section{CASO CLÍNICO}

Paciente de 40 años de edad, sexo masculino, con antecedente de depresión en tratamiento con 
psicoterapia. Consultó con cuadro de un mes de compromiso del estado general. Una semana antes de consultar presentó dolor en hipocondrio derecho, intermitente, que no cedía con la ingesta de analgésicos. Tres días antes de consultar presentó fiebre no cuantificada.

En la evaluación inicial destacaba frecuencia cardíaca de $110 \mathrm{~lx}^{\prime}$, frecuencia respiratoria de 29 respiraciones/minuto, temperatura axilar de $38,5^{\circ} \mathrm{C}$, murmullo disminuido en la base pulmonar derecha y dolor a la palpación en hipocondrio derecho. Los exámenes de laboratorio mostraron: leucocitos 12.000 células $/ \mathrm{mm}^{3}$, con neutrófilos de $78 \%$ y baciliformes de 1\%; hemoglobina $12,6 \mathrm{~g} / \mathrm{dl}$; velocidad de eritrosedimentación $85 \mathrm{~mm} / \mathrm{h}$; proteína $\mathrm{C}$ reactiva (PCR) $239 \mathrm{mg} / \mathrm{dl}$; bilirrubina total $0,34 \mathrm{mg} /$ $\mathrm{dl}$; bilimubina directa $0,11 \mathrm{mg} / \mathrm{dl}$; fosfatasa alcalina $151 \mathrm{U} / \mathrm{L}$; tiempo de protrombina 71,9\%; amilasa 44 $\mathrm{U} / \mathrm{L}$; lipasa $116 \mathrm{U} / \mathrm{L}$

Se realizó ecotomografía abdominal que mostró una lesión de márgenes irregulares predominantemente hipoecogénica, con zonas hiperecoicas en su interior, de $14 \mathrm{~cm} \mathrm{x} 7 \mathrm{~cm}$, ubicada en el lóbulo hepático derecho (Sg6 y Sg7) (Figura 1). Se solicitó tomografía computarizada (TAC) de abdomen que mostró una imagen predominantemente hipodensa, de bordes imegulares en Sg7 y Sg8, con leve impregnación periférica posterior al uso de medio de contraste endovenoso, la cual se acentúa discretamente en fase tardía, sin evidenciarse otras lesiones (Figura 2).

Otros exámenes de laboratorio mostraron antígeno carcinoembrionario (ACE), $0,6 \mathrm{ng} / \mathrm{ml}$; alfa feto proteína (AFP), 0,87 ng/ml, CA-19,9, 0,15 U/ml; anticuerpos IgM para hepatitis A, negativos; antígeno de superficie de virus de hepatitis $B$, negativo; anticuerpos anti-hepatitis $\mathrm{C}$, negativos; anticuerpos IgG e IgE para hidatidosis, negativos; anticuerpos IgG para amebiasis, negativos.

Por persistencia de signos de respuesta inflamatoria sistémica y con sospecha de absceso hepático, fue llevado $48 \mathrm{~h}$ después de su ingreso a drenaje guiado por TAC de abdomen, donde se obtuvieron $150 \mathrm{ml}$ de líquido hemorrágico, mostrando colapso prácticamente completo de la imagen en TAC de
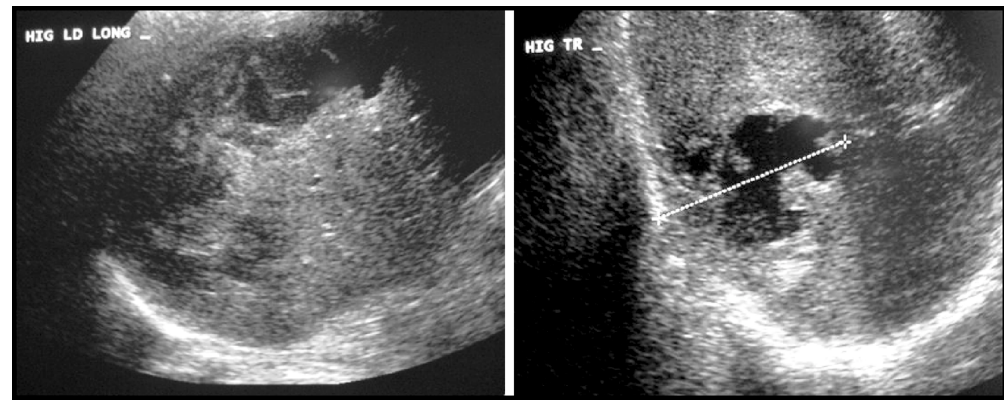

Figura 1. Ecotomografía hepática, que demuestra lesión focal heterogénea de predominio anecogénica, con áreas ecogénicas en su interior y márgenes irregulares.

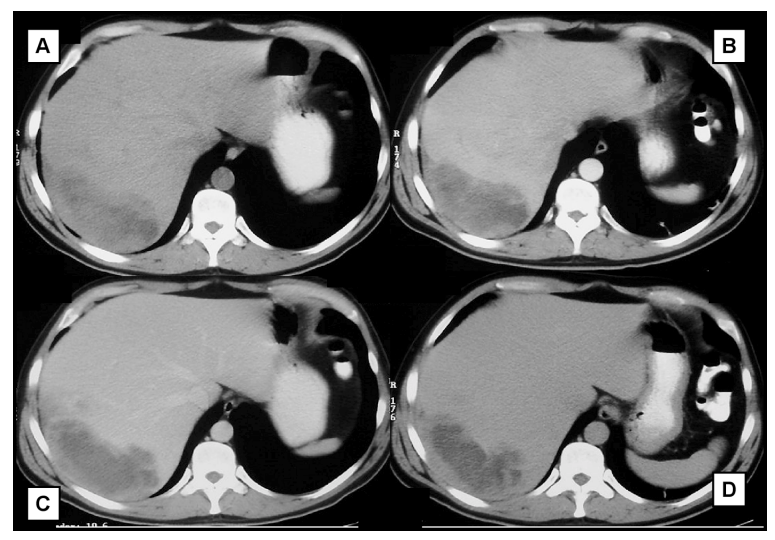

Figura 2. TAC región hepática. (A) Sin contraste EV, muestra lesión focal heterogénea, hipodensa con márgenes gruesos, irregulares. (B), (C) Fases arterial y venosa, muestran impregnación periférica en forma irregular, permaneciendo región central hipodensa. (D) Fase tardía, mayor impregnación periférica, con presencia de algunos finos tabiques internos. 
control. El estudio del material drenado mostró al análisis citoquímico: líquido predominantemente hemorrágico, Gram y cultivo negativos y se consideró la muestra inadecuada para citología.

El paciente persistió con signos de respuesta inflamatoria sistémica, se realizó un TAC abdominal de control que mostró reproducción completa de la lesión hepática. Se decidió llevar a drenaje por laparotomía, donde se encontró cavidad en Sg6 y Sg7, con contenido hemático en su interior, adherida al peritoneo parietal y diafragma. No se encontraron otras lesiones, ni adenopatías abdominales.

Se tomó biopsia contemporánea de la lesión que mostró tejido fibroconectivo en partes extensamente necrótico; reconociéndose algunas células con atipias celulares. La biopsia diferida mostró un tumor maligno indiferenciado infiltrante en tejido hepático, sin presentar elementos sarcomatoides.

El estudio inmunohistoquímico, con técnica de estreptavidina biotina (Zimed), concluyó un hepatocarcinoma poco diferenciado, con positividad para citoqueratinas de alto y bajo peso molecular, vimentina y AFP y ausencia de reactividad para ACE, CD30 y fosfatasa alcalina placentaria.

Se realizó gastroscopia, colonoscopia, TAC de tórax, TAC de abdomen y pelvis, y ecotomografía testicular que no evidenciaron nuevas lesiones.

Se decidió realizar una nueva exploración quirúrgica, con intención de realizar una resección hepática. Se encontró una lesión en Sg7 y Sg8, con infiltración del diafragma y peritoneo parietal, sin otras lesiones hepáticas, ni adenopatías. Se realizó hepatectomía derecha, con resección parcial de diafragma y reparo primario.
La biopsia diferida incluía lóbulo derecho hepático con gran lesión tumoral expansiva de 11,5 por $7,5 \mathrm{~cm}$ de diámetros mayores, de disposición subcapsular, en partes extensamente necrótica y cavitada, sin evidencias de cirrosis en el tejido hepático subyacente (Figura 3).

Microscópicamente el tumor estaba compuesto predominantemente por un componente epitelial con escasas áreas sarcomatoides. El componente epitelial presentaba células con moderada cantidad de citoplasma y núcleos aumentados de tamaño con cromatina granular gruesa y mitosis frecuentes. El componente sarcomatoide se apreciaba de aspecto fusado pleomórfico con nucleolo conspicuo, alternando con áreas de necrosis y formación de tejido granulatorio (Figura 4), El examen inmunohistoquímico realizado con técnica de estreptavidina biotina (Zimed), mostró intensa reactividad en las células tumorales para ACE, CD10 y citoqueratina 7, sin reactividad para AFP en las áreas sarcomatoides (Figura 5). Concluyendo el diagnóstico de hepatocarcinoma poco diferenciado variedad sarcomatoide.

En el periodo postoperatorio se presentó disfunción hepática transitoria, que se resolvió al séptimo día postoperatorio y un síndrome vertiginoso al quinto día postoperatorio, que se estudió con TAC y resonancia magnética nuclear (RMN) cerebral, concluyéndose de ongen penférico y resolviéndose al décimo cuarto día postoperatorio. Fue dado de alta en buenas condiciones al décimo sexto día postoperatorio.

A los 20 días del periodo postoperatorio se efectuó el primer control, estando asintomático, con TAC abdominal de control sin evidencia de recumencia.
Figura 3. Las imágenes macroscópicas de la pieza quirúrgica de lobectomía derecha (A) y durante el procesamiento en secciones sagitales (B), en los que se observa una gran masa tumoral que compromete bordes quinúrgicos de sección y en el cual se reconoce cavitación con áreas de necrosis, hemorragia y abscedación.

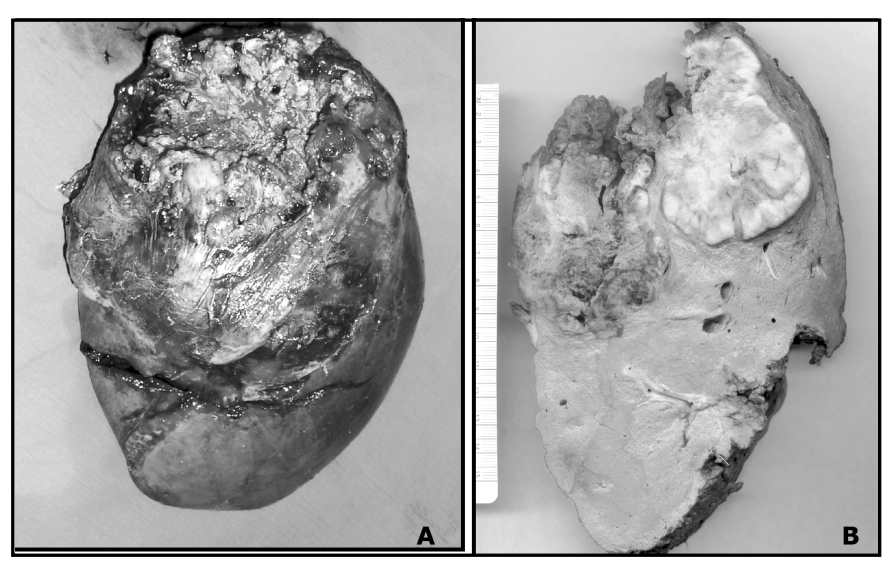




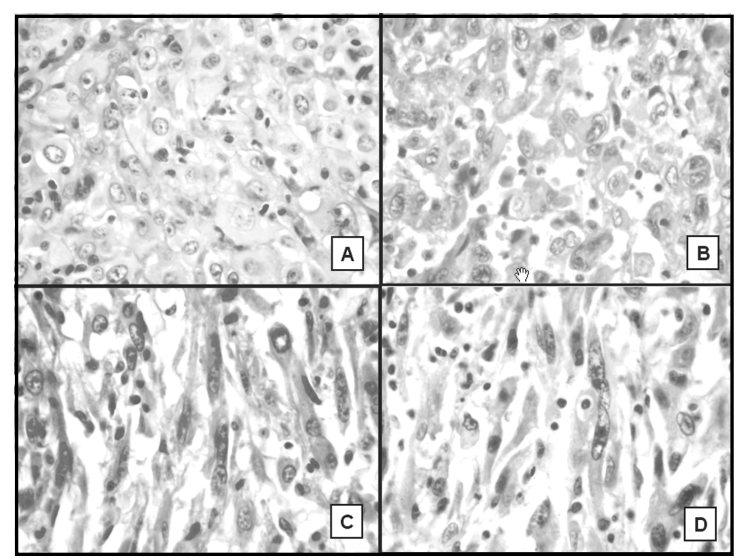

Figura 4. Microscópicamente el tumor presentaba áreas heterogéneas. (A) Se observa patrón clásico de hepatocarcinoma con células redondeadas de límites precisos con núcleos que presentan nucleolos prominentes acompañado de escasos PMN. (B) El mismo patrón pero con leve retracción citoplasmática y desprendimiento celular. (C) y (D) Las células tumorales se observan fusadas, alargadas con núcleos aumentados de tamaños algunos con características bizarras y nucleolos prominentes y mitosis presentes.

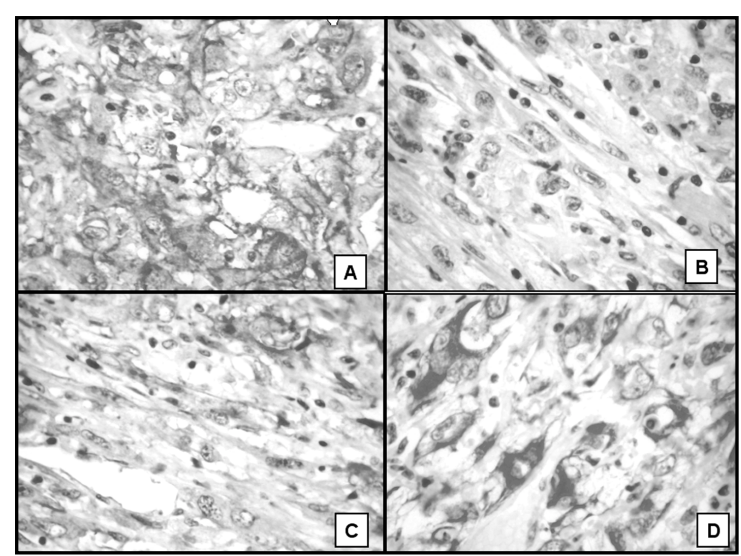

Figura 5. Estudio inmunohistoquímico mostró intensa positividad para AFP en áreas epiteliales (A) y ausencia de expresión en áreas sarcomatosas (B), la vimentina se expresó positivamente en el componente epitelial y no epitelial (C), las citoqueratinas de alto y bajo peso molecular se observaron exclusivamente en las áreas epiteliales (D).

\section{DisCUSIÓN}

El HCTS es poco frecuente, las estadísticas japonesas muestran una prevalencia de $1,8 \%$ de los hepatocarcinomas resecados quirúrgicamente ${ }^{6}$. El dolor abdominal y la fiebre son síntomas frecuentes, simulando un absceso hepático ${ }^{1,2}$.

En las comunicaciones publicadas se encuentra una frecuencia baja de cirrosis hepática asociada y un nivel bajo o negativo de $\mathrm{AFP}^{7}$. El presente caso se presentó con dolor en hipocondrio derecho, fiebre y leucocitosis; los niveles de AFP fueron negativos y las células tumorales mostraron una reacción negativa a AFP; sin evidencia de cirrosis hepática en la pieza de patología.

Con respecto al estudio imagenológico, un trabajo reciente de 34 hepatocarcinomas resecados mostró que seis (18\%) fueron diagnosticados por las imágenes de TAC abdominal como lesiones benignas (abscesos hepáticos) u otras lesiones neoplásicas (tumor de Klatskin, metástasis de cáncer colorrectal y cáncer de vesícula biliar). Todos los pacientes tenían niveles normales de $\mathrm{AFP}^{8}$.

La patogénesis de la transformación sarcomatoide del carcinoma hepático no ha sido clarificada. Sin embargo, la evidencia sugiere que las células fusadas del hepatocarcinoma representan una diferenciación sarcomatosa de las células epiteliales más que una combinación de sarcoma y hepatocarcinoma ${ }^{1}$.

El mecanismo de degeneración, necrosis y regeneración de las células de carcinoma debidas a fármacos antineoplásicos o quimioembolización trans-arterial, han sido postulados como posibles inductores en otros casos ${ }^{1,6}$.

Los trabajos previos muestran combinaciones con componentes condrosarcomatosos ${ }^{9-11}$, rabdomiosarcomatosos ${ }^{12,13}$ y con presencia de células gigantes tipo osteoclasto ${ }^{14,15}$.

Los hepatocarcinomas en general, se desarrollan en hígados previamente dañados con cirrosis post virales y de tipo nutricio-alcohólico. En el caso de los hepatocarcinomas con componente sarcomatoide, la literatura no muestra una tendencia única, pues se pueden encontrar comunicaciones de estos tumores desde hígados con cirrosis post hepatitis $\mathrm{B}^{12}$, cirrosis biliar primaria ${ }^{16}$ o en hígados sin alteraciones previas ${ }^{13}$.

Inmunohistoquímicamente existe alta variabilidad en los patrones de expresión, dependiendo lógicamente en el tipo de diferenciación sarcomatosa, sin embargo, lo más frecuente es encontrar constantemente positividad para $\operatorname{AFP}^{7,9,12,17}$ en el componente epitelial y negatividad en el componente sarcomatoso, tal como ocurrió en este caso. 
La coexistencia de algunos marcadores, tanto en el componente epitelial como el componente sarcomatoso, ha sido el argumento de algunos autores para sugerir que el componente sarcomatoso de esos tumores deriva de células hepáticas propiamente tal y no del componente mesenquimático estromal hepático ${ }^{18}$.

\section{REFERENCIAS}

1. Eriguchi N, Aoyagi S, Okuda K, Hara M, Fukuda S, TAMAE $T$ ET al. Unusual liver carcinomas with sarcomatous features: analysis of four cases. Surg Today 2001; 31: 530-3.

2. Okuda K, Kondo Y, Nakano M, Kage M, Arakawa M, KoJIRO M ET AL. Hepatocellular carcinoma presenting with pyrexia and leukocytosis: report of five cases. Hepatology 1991; 13: 695-700.

3. STEINER PE. Cancer of liver and cirrhosis in transSaharan Africa and the United States of America. Cancer 1960; 13: 1085-166.

4. Окuda K, Peters RL, Simson IW. Gross anatomical features of hapatocellular carcinoma from three disparate geographic areas: proposal a new classification. Cancer 1984; 54: 2165-73.

5. StrasBerg SM. Nomenclature of hepatic anatomy and resections: a review of Brisbane 2000 system. J Hepatobiliary Pancreat Surg 2005; 12: 351-5.

6. Han JH, Park YN, Jung WH, Chi HS, Park C. A case with sarcomatoid hepatocellular carcinoma. Yonsei Med J 1998; 39: 390-4.

7. KaziKoe S, Kojiro M, NaKashima T. Hepatocellular carcinoma with sarcomatous change. Clinicopathologic and inmunohistochemical studies of 14 autopsy cases. Cancer 1987; 59: 310-16.

8. Chung FA, Thng CH, Lui HF, Mancer K, Chow PKH, TAN YM ET AL. Clinical mimicry of hepatocellular carcinoma: imaging-pathological correlation. Singapore Med J 2005; 46: 31-37.

9. Fu Y, Kobayashi S, Kushida Y, Saoo K, Haba R, Mori S ET AL. Sarcomatoid hepatocellular carcinoma with chondroid variant: case report with immunohistochemical findings. Pathol Int 2000; 50: 919-22.

10. IKebe $T$, WaKasa $K$, Sasaki M, Hamba $H$, KaneKo $M$, Y АмAмото T ET AL. Hepatocellular carcinoma with
Los trabajos previos muestran que la sobrevida de los pacientes con HCTS es menor que los pacientes con HCC, lo cual puede ser debido a la agresiva diseminación intrahepática y la alta frecuencia de metástasis ${ }^{1,6}$. En el estudio del presente caso no se evidenciaron signos de diseminación intrahepática, ni metástasis durante la intervención quirúrgica.

chondrosarcomatous variation: case report with immunohistochemical findings, and review of the literature. J Hepatobiliary Pancreat Surg 1998; 2: 217-20.

11. Ooi A, Katsuda S, Nakanishi I, Nakamura M, Matsushita F, TanaKa $N$ et aL. Hepatocellular carcinoma with chondrosarcomatous variation. A case report and review of the literature. Acta Pathol Jpn 1987; 37: 1165-73.

12. Akasofu M, Kanahara E, Kaji K, Nakanishi I. Sarcomatoid hepatocellular-carcinoma showing rhabdomyoblastic differentiation in the adult cirrhotic liver. Virchows Arch 1999; 434: 511-15.

13. Сно MS, Lee SN, Sung SH, Han WS. Sarcomatoid hepatocellular carcinoma with hepatoblastoma-like features in an adult. Pathol Int 2004; 54: 446-50.

14. IkEda T, Seki S, Maki M, Noguchi N, Kanamura T, Ari S ET AL. Hepatocellular carcinoma with osteoclastlike giant cells: possibility of osteoclastogenesis by hepatocyte-derived cells. Pathol Int 2003; 53: 450-6.

15. Sasaki A, Yokoyama S, NaKayama I, NaKashima K, Kim YI, KITANO S. Sarcomatoid hepatocellular carcinoma with osteoclast-like giant cells: case report and immunohistochemical observations. Pathol Int 1997; 47: 318-24.

16. Komada N, Yamagata M, Komura K, Hayashi K, MaruYama T, KataOKa H et al. Hepatocellular carcinoma with sarcomatous change arising in primary biliary cirrhosis. J Gastroenterol 1997; 32: 95-101.

17. Haratake J, Horie A. An inmunohistochemical study of sarcomatoid liver carcinomas. Cancer 1991; 68: 93-7.

18. Oda Y, Katsuda S, NaKanishi I. An autopsy case of hepatic sarcomatoid tumor: immunohistochemical comparison with a sarcomatous component of hepatocellular carcinoma. Pathol Int 1994; 44: 230-6. 